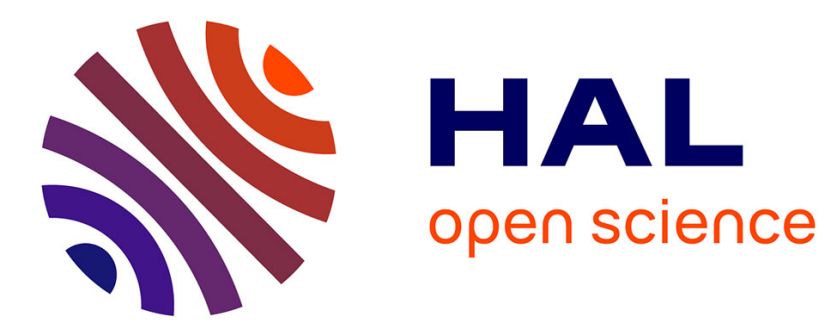

\title{
Analyser l'usage didactique d'un EIAH en mathématiques : une tâche nécessairement complexe
}

Dominique Guin, Luc Trouche

\section{To cite this version:}

Dominique Guin, Luc Trouche. Analyser l'usage didactique d'un EIAH en mathématiques: une tâche nécessairement complexe. Sciences et Techniques Educatives, 2001, 8(1/2), pp.61-74. hal-00190084

\section{HAL Id: hal-00190084 \\ https://telearn.archives-ouvertes.fr/hal-00190084}

Submitted on 23 Nov 2007

HAL is a multi-disciplinary open access archive for the deposit and dissemination of scientific research documents, whether they are published or not. The documents may come from teaching and research institutions in France or abroad, or from public or private research centers.
L'archive ouverte pluridisciplinaire HAL, est destinée au dépôt et à la diffusion de documents scientifiques de niveau recherche, publiés ou non, émanant des établissements d'enseignement et de recherche français ou étrangers, des laboratoires publics ou privés. 


\title{
Analyser l'usage didactique d'un EIAH en mathématiques : une tâche nécessairement complexe
}

\author{
Dominique Guin et Luc Trouche \\ Laboratoire ERES, \\ Université Montpellier 2 \\ Département de Mathématiques - CC 051 \\ Place Eugène Bataillon \\ 34095 Montpellier Cedex5 \\ \{guin,trouche\}@math.univ-montp2.fr
}

RÉSUMÉ. Dans cet article, nous nous intéressons à l'analyse de l'usage des EIAH dans l'enseignement des mathématiques. Dans une première partie, nous décrivons l'évolution des problématiques de travaux portant sur ce thème depuis une dizaine d'années. Après une phase de recherches basées, pour la plupart d'entre elles, sur un postulat d'amélioration de l'apprentissage, l'identification de certains phénomènes didactiques a conduit au développement de nouvelles approches théoriques permettant de prendre en compte ces phénomènes. A l'aide d'exemples, nous introduisons succinctement les approches instrumentale et écologique. La deuxième partie présente une recherche de plusieurs équipes visant à étudier les travaux existants pour dégager une démarche d'analyse de l'intégration des EIAH dans l'enseignement des mathématiques. Cette étude confirme la nécessité de diversifier les approches pour appréhender la complexité de l'usage didactique d'un EIAH. Nous décrivons brièvement la méthodologie et les résultats de cette recherche en relation avec les phénomènes décrits précédemment.

ABSTRACT. In this paper, we are interested in the analysis of the instructional use of mathematical ILE. First, we will describe the evolution of "problematics » in research studies dealing with this subject over the last ten years. After a period of studies mostly based on a postulate of learning improvement, some didactical phenomena were highlighted. This has led to the development of theoretical approaches which allow to take into acccount these phenomena. We will introduce the instrumental and ecological approaches with examples. Secondly, we will present a collaborative research that aims to underline processes for analysing studies concerning the integration of ILE into mathematics teaching. This study confirms the necessity of varying approaches in order to grasp the complexity of the instructional use of an ILE. We will briefly describe the methodology and the main results in relation to the phenomena previously mentioned.

MOTS-CLÉS : EIAH, usage didactique, instrument, viabilité, écologie, intégration, contraintes.

KEY WORDS: ILE, instructional use, instrument, viability, ecology, integration, constraints. 


\section{Introduction}

L'enseignement des mathématiques peut apparaître comme un domaine privilégié de l'intégration des EIAH, pour au moins deux raisons :

- une grande diversité des EIAH disponibles, sur le plan des logiciels (logiciels de géométrie dédiés à l'enseignement, logiciels de calcul formel, didacticiels) comme sur le plan des matériels (calculatrices ou ordinateurs);

- une proximité des disciplines mathématique et informatique, avec des interactions dans les domaines de la recherche et des pratiques professionnelles.

Or, malgré une volonté institutionnelle affirmée à l'occasion de chaque changement de programme depuis une vingtaine d'années ${ }^{1}$, cette intégration demeure très faible ${ }^{2}$. Le paragraphe suivant nous aide à comprendre ce phénomène.

\section{L'évolution des recherches}

\subsection{D'un discours très optimiste à un discours plus circonspect}

S'appuyant sur un rapport positif des élèves aux EIAH [ART 95] et sur les potentialités des nouveaux outils, les premiers discours sont très optimistes. Dans une synthèse des recherches sur les calculatrices graphiques [PEN 96], les auteurs notent ainsi : "Many authors believe that graphics calculators have the potential to revolutionalise mathematics education, both in the way it is taught and the content and emphases of curricula. Consequently, there has been much enthusiastic comment concerning the capabilities, potential and implications of this technology ».

Ces recherches, sous l'influence dominante du constructivisme piagétien, sont marquées par une double illusion:

- l'illusion d'un apport naturellement positif pour l'apprentissage: l'environnement permet de voir, donc de comprendre ;

\footnotetext{
${ }^{1}$ Le programme de seconde, applicable à la rentrée 2000, précise : « Il est nécessaire de revisiter l'enseignement des mathématiques à la lumière des immenses possibilités offertes (...) ; l'usage éclairé d'outils informatiques est donc recommandé dans chaque partie du programme ».

${ }^{2}$ Le rapport annuel 2000 de l'IGEN (Inspection Générale de l'Education Nationale) relève « un clivage entre une minorité d'enseignants motivés fortement utilisateurs, développant des utilisations et des productions de qualité, et une majorité non encore investie ».
} 
- l'illusion d'un apport positif pour l'enseignement : l'environnement, prenant en charge la partie technique de la tâche, permet une centration sur la partie conceptuelle [KUT 94].

Les discours semblent évoluer au cours des années 90 avec les premières analyses. [BAR 91] précise que la mise en œuvre des EIAO avait lieu, à cette époque, dans des conditions expérimentales très exceptionnelles (généralement en dehors des activités traditionnelles d'une classe) ; cependant ces premières analyses a priori ont permis de mettre en évidence certains problèmes didactiques. Ainsi, l'analyse de plusieurs logiciels d'aide à la démonstration géométrique réalisée avec des enseignants dans [GUI 91 \& 95] a montré qu'un logiciel tel que Geometry Proof Tutor [AND 87], conçu pour valider une théorie cognitive ACT, peut s'avérer inutilisable dans un contexte didactique, les choix cognitifs et didactiques sousjacents étant en contradiction avec ceux d'un système éducatif donné. De plus, ce résultat ne se limite ni au domaine de la géométrie, ni à un système éducatif spécifique : [AND 92] constate, lui aussi, que l'introduction dans les classes d'un logiciel d'algèbre pose des problèmes, les techniques accessibles à l'interface n'étant pas conformes à celles que les enseignants voudraient utiliser ${ }^{3}$.

\subsection{Identification de phénomènes conduisant aux premiers questionnements sur le postulat [PEN 96] d'amélioration de l'apprentissage}

[BRU 94] relèvent aussi l'importance des situations d'apprentissage, « les outils n'ayant de sens que par rapport aux situations dans lesquelles ils sont mis en œuvre ». C'est d'ailleurs la multiplication des observations dans un contexte d'enseignement standard (ni en soutien, ni en laboratoire) qui met en évidence des difficultés que le seul cadre théorique piagétien ne permet pas d'analyser. La problématique de l'intégration de l'informatique dans l'enseignement apparaît explicitement dans [BAR 96] qui analysent les différents modes d'usage de l'informatique dans l'éducation ; simultanément, [BRU 97] retrace l'évolution des rapports entre informatique et éducation, à travers les potentialités et les difficultés, en particulier celles des tuteurs intelligents où il souligne le rôle déterminant de l'interface.

Les premières recherches sur l'intégration du logiciel Derive, menées en France dans un cadre institutionnel à partir de 1991, révèlent des phénomènes didactiques liés à la transposition informatique décrite par [BAL 94-1] comme « ce travail sur la connaissance qui en permet une représentation symbolique et la mise en œuvre de cette représentation par un dispositif informatique ». Par conséquent, il faut déterminer le domaine de validité épistémologique de l'EIAO [BAL 94-2], c'est-àdire caractériser les problèmes auxquels il donne accès, identifier ses

${ }^{3}$ Les travaux de cette équipe ont évolué : les auteurs décrivent dans [KOE 97] une expérimentation d'intégration d'un EIAH dans les classes où enseignants et élèves sont enthousiastes. 
caractéristiques sémiotiques et fonctionnelles, ainsi que la cohérence interne et la tolérance du dispositif.

[ART 95] relève :

- le phénomène de pseudo transparence lié à l'écart entre ce qui est écrit par l'élève et ce qui est affiché sur l'écran :

[Pour entrer $(a+2) / 5]$ « certains élèves, ayant correctement rajouté un couple de parenthèses autour de $(\mathrm{a}+2)$, s'étonnent d'avoir un affichage sans parenthèses et se demandent si leur production est correcte ou non. L'apparition et la disparition des parenthèses paraît obéir pour certains à un jeu mystérieux qu'ils comprennent d'autant moins que justement ils ne maîtrisent pas le parenthésage ».

- le phénomène de double référence lié à la confrontation de l'environnement papier-crayon et de l'EIAH. [ART 95] évoque ainsi la factorisation rationnelle de $\mathrm{x}^{\mathrm{n}}-1$ en classe de $1^{\text {ère }}$ :

« dans l'environnement papier/crayon, la factorisation des polynômes est reliée, à ce niveau d'enseignement, à la recherche de racines réelles (...). L'algorithmisation Derive dans l'univers interne de la machine passe, elle, par des factorisations intermédiaires dans $\mathrm{Z} / \mathrm{pZ}$ si $\mathrm{n}=2 \mathrm{p}(\ldots)$ : la factorisation par $(\mathrm{x}-1)$ par exemple n'est obtenue que pour $\mathrm{n}$ premier et la factorisation par $(\mathrm{x}-1) .(\mathrm{x}+1)$ que pour $\mathrm{n}=4(\ldots)$. Les élèves qui se situent dans la deuxième interprétation ont beaucoup plus de difficultés à produire des conjectures ».

D'autres études mettent en évidence que les processus d'adaptation à l'environnement ne produisent pas nécessairement les connaissances attendues :

- [DAG 96] montre que l'usage régulier d'un logiciel (Fonctuse) ne suffit pas à garantir la construction, par les élèves, de l'articulation entre les registres algébrique et graphique ;

- [TRO 96] montre la confusion possible entre l'objet et ses représentations : la réponse à la question « la fonction $\mathrm{f}$ définie par $f(x)=\ln x+10 \sin x$ admet-elle pour limite $+\infty$ en $+\infty$ ? » dépend fortement de l'environnement. Si les élèves disposent d'un grapheur, la représentation graphique obtenue (cf. figure 1) induit, du fait de l'oscillation, une réponse fausse dans $25 \%$ des cas ( $5 \%$ de réponses fausses sans calculatrice). 


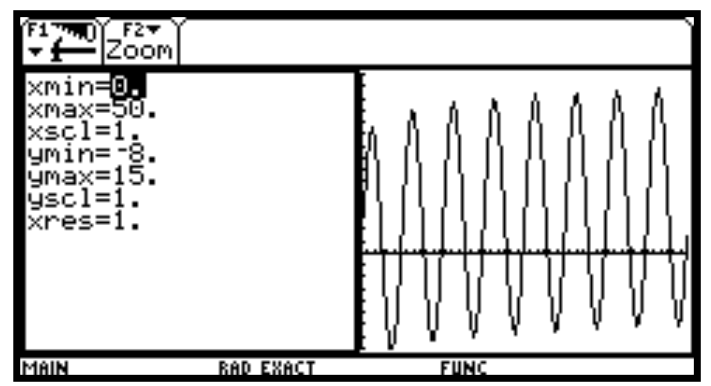

Figure 1. Une représentation graphique de la fonction $x \mapsto \ln x+10 \sin x$

Cet exemple remet en question l'assertion que les possibilités de visualisation conduisent nécessairement à une meilleure compréhension des concepts. De plus, [LUE 98] remarquent qu'il est difficile d'évaluer les rapports entre les performances observées et la nature des connaissances construites. Est-ce le témoignage d'une bonne maîtrise de l'environnement ou le témoignage d'un progrès de l'apprentissage ? Il est donc indispensable d'identifier les contraintes informatiques des EIAH relativement d'une part au contenu, et d'autre part à la gestion de l'interaction entre élève et machine, pour connaître précisément les solutions que l'élève peut construire. En outre, [PEN 96] remarquent que les recherches ont négligé jusqu'alors la disponibilité de l'outil et la familiarité des élèves avec ces outils. Ces différents facteurs vont être pris en compte dans de nouvelles approches.

\section{De nouveaux cadres pour analyser ces phénomènes}

L'attention portée aux médiations nécessaires à tout processus d'apprentissage suscite l'apparition de nouvelles approches théoriques, relatives au rôle que jouent les instruments matériels et symboliques de l'activité mathématique ou aux rapports institutionnels dans lesquels s'inscrivent les apprentissages.

\subsection{Approche instrumentale}

La transposition informatique et les choix des concepteurs produisent dans un EIAH des contraintes que [TRO 00] classe en contraintes internes, de commande et d'organisation. Elles contraignent, de façon relative, non seulement les modes d'action, mais également les modes de pensée de l'utilisateur. [RAB 99] distingue l'outil technique ( « l'artefact »), qui est donné, et l'instrument qui est construit par le sujet. Cette construction, ou genèse instrumentale, est liée aux caractéristiques de l'artefact (ses potentialités et ses contraintes) et du sujet (ses connaissances et ses habitudes de travail antérieures). Elle combine deux processus : 
- un processus d'instrumentalisation, processus d'enrichissement des propriétés de l'artefact par le sujet ; [TRO 98] évoque différentes transformations des outils par les élèves d'une classe expérimentale : modification de la barre des menus, création de raccourcis de clavier, stockage de programmes dédiés etc. ;

- un processus d'instrumentation, défini par l'accommodation des schèmes d'un sujet, i.e. des invariants dans sa conduite, pour la réalisation d'une tâche donnée [VER 96] ; [DEF 00] décrit ainsi, à propos du réglage de la fenêtre permettant de voir la courbe d'une fonction sur l'écran d'une calculatrice, l'évolution de ce processus chez un élève, en relation avec les potentialités et les contraintes de l'outil (des commandes plus nombreuses sont mobilisées, de façon plus pertinente, avec un recours mieux maîtrisé au papier/crayon).

Ces processus ne sont pas homogènes : le suivi de la genèse instrumentale dans une classe permet à [TRO 00] de repérer des régularités dans les comportements d'un individu et de constituer une typologie des comportements. La dispersion des comportements dans la classe apparaît bien sûr liée aux connaissances mathématiques, mais aussi aux métaconnaissances [BAR 93] construites par les élèves dans la gestion des contraintes de l'outil et des situations.

\subsection{Approche écologique}

[CHE 96] décrit la constitution d'une organisation mathématique autour de la réalisation de tâches, appelant la création de techniques, justifiées par des technologies, développées dans le cadre d'une théorie. Il analyse les pratiques mathématiques en termes de rapports que les élèves établissent avec ces organisations, dans le cadre d'une institution : dans une institution donnée, des techniques officielles sont introduites, aboutissant à la routinisation de certaines tâches et à l'affaiblissement des technologies qui les accompagnaient.

Cette approche écologique avait déjà amené [CHE 92] à poser le problème de la viabilité des objets informatiques dans l'enseignement. Elle conduit à l'analyse des nouveaux outils introduits dans la classe [ART 99], des rapports entre techniques habituelles et techniques nouvelles [LAG 99-1] et débouche sur la nécessité de socialiser les processus d'instrumentation, dans le cadre d'une organisation particulière du temps et de l'espace scolaires [GUI 99]. Cette démarche impose de concevoir de nouveaux dispositifs dans la classe et d'étudier leur viabilité [GUI 00]. De plus, le rôle de l'enseignant, dont l'importance est déjà soulignée dans [VIV 90], s'avère véritablement crucial dans la réorganisation des processus d'enseignement, en particulier dans la décontextualisation des connaissances acquises dans l'EIAH.

Une attention particulière est portée dans ce cadre aux choix des situations qui permettent de gérer les contraintes inévitables de l'outil et de tirer parti de ses potentialités, confirmant le point de vue de [BRU 94] : « l'efficacité d'un instrument 
n'est pas tant due à ses caractéristiques techniques qu'à sa pertinence relative à la fonction qu'il assure dans la situation où il intervient ». Plus précisément, [LAB 99] identifie, comme indice d'intégration, le fait que l'EIAH ne soit pas utilisé à des seules fins d'illustration, mais soit un constituant essentiel de l'activité (l'EIAH étant le seul moyen de la réaliser ou permettant des stratégies de résolution différentes).

\subsection{La question des ostensifs}

La distinction entre ostensifs ${ }^{4}$ et non ostensifs [BOS 99] permet un approfondissement des approches instrumentale et écologique :

- [BOS 99] mettent en évidence l'instrumentalité d'un ostensif, dépendant du nombre de techniques dans lesquelles cet ostensif intervient et du caractère robuste et fiable de ces techniques dans l'accomplissement des tâches en question : de ce point de vue, l'instrumentalité de l'ostensif $x^{1 / 2}$ est plus grande que celle de l'ostensif $\sqrt{x}$. L'étude des ostensifs des environnements papier-crayon et de ceux des environnements informatiques, avec leurs instrumentalités respectives, permettent d'appréhender plus précisément la manière dont les EIAH contraignent les modes d'action et de pensée. [DEF 00] relève ainsi que l'ostensif "Solve", disponible comme commande sur une calculatrice, permet une évolution du rapport de l'élève à l'objet «équation du second degré »: dans un environnement papier/crayon, ce type d'équation apparaît comme relevant d'une technique spécifique (« le calcul d'un discriminant»), alors que, dans un environnement calculatrice, elle apparaît comme élément d'un ensemble plus vaste, celui des équations numériques ;

- [LAG 99-2] montre que cette distinction ostensifs/non-ostensifs, à chacun des niveaux de la trichotomie technique/technologie/théorie, permet de se dégager d'un point de vue naïf : la conceptualisation n'est pas un processus à sens unique entre un niveau « matériel », contingent, et un niveau « noble », celui de la pensée et des concepts.

Ces nouvelles approches donnent de nouveaux outils pour analyser les travaux relatifs à l'intégration des EIAH.

\section{Une démarche d'analyse de l'intégration des EIAH}

\subsection{Problématique}

\footnotetext{
${ }^{4}$ Les ostensifs sont, parmi les objets reconnus d'une activité mathématique, ceux qui sont perceptibles et manipulables (le symbole $\sqrt{ }$ par exemple) ; ils sont inséparables, dans l'activité mathématique, des non-ostensifs, objets non tangibles qui guident leur manipulation (la propriété de croissance d'une fonction par exemple).
} 
Cette seconde partie présente partiellement la recherche menée, sous la direction de Michèle Artigue, avec la collaboration des laboratoires DIDIREM, LEIBNIZIMAG, LIUM, ERES et de l'IUFM de Bretagne. Cette recherche s'est effectuée dans le cadre d'une réponse à l'appel d'offres du CNCRE (Ministère de l'Education Nationale) portant sur les questions suivantes :

"Comment les TIC sont-elles utilisées dans le système éducatif? Modifient-elles la nature, les contenus et les modalités des apprentissages, ainsi que les acquis, le rapport au savoir et les attitudes des élèves, des étudiants et des enseignants?".

L'objectif de cette recherche était d'analyser un vaste corpus de travaux récents portant sur l'intégration des EIAH dans l'enseignement, pour essayer de comprendre les raisons du décalage évoqué précédemment entre le discours optimiste de la plupart des articles et l'intégration marginale constatée dans de nombreux pays. Il s'agissait donc d'analyser leur problématique, cadre théorique et méthodologie et de discuter les apports et limites de leurs résultats dans une perspective d'intégration.

\subsection{Présentation de la méthodologie}

La première phase avait pour objectif de constituer un large corpus de publications, susceptible de rendre compte de la diversité des travaux portant sur les EIAH et de leur exploitation dans l'enseignement des mathématiques, aux niveaux national et international, durant la période 1994-1999. Cette diversité pouvait porter aussi bien sur les environnements concernés, les problématiques et approches théoriques que sur les types de recherches (conception ou évaluation, expérimentation à différents niveaux de formation etc.). Le premier corpus de 662 articles a été constitué à partir d'un ensemble de revues représentatives : Revue Française de Pédagogie, Recherches en Didactique des Mathématiques, Educational Studies in Mathematics, Sciences et Techniques Educatives, Journal of Artificial Intelligence in Education, actes des colloques EIAO, revues INRP, IREM etc. Un second corpus restreint de 79 articles a été sélectionné en fonction de la représentativité de l'article dans sa catégorie ou de l'intérêt qu'il constituait pour notre problématique $(\$ 4.1)$.

Les premières analyses qualitatives nous ont permis de mettre en évidence la grande diversité des approches et nous ont conduits à constituer une grille d'analyse pour rechercher une cohérence et rendre compte, pour chacune d'elle, des résultats essentiels. Les questions de cette grille d'analyse (qui comporte 96 items) relèvent des domaines de l'ergonomie et de la psychologie cognitives, des sciences de l'éducation, de la didactique des mathématiques et de l'interaction homme-machine (IHM). Une partie des questions portait sur l'analyse des EIAH, que ce soit du point de vue de la transposition informatique, des potentialités et contraintes de l'instrument ou des caractéristiques de l'IHM (navigation, interaction et prise en compte de l'utilisateur). Deux autres parties concernaient, d'une part l'analyse des 
représentations des élèves et enseignants vis à vis de ces environnements et, d'autre part, l'analyse de l'impact de ces environnements sur les processus d'apprentissage et sur le rapport au savoir visé par l'institution. La dernière partie des questions était relative à l'analyse des situations d'enseignement intégrant des EIAH et des pratiques des enseignants. Cette grille a été exploitée pour opérer des regroupements permettant de distinguer des approches différentes de l'intégration. Le traitement a été effectué sur l'ensemble des items, puis pour ceux relatifs à chaque approche. Au sein de chaque classe, les traitements statistiques ont permis de déterminer les articles centraux et de préciser les modalités caractéristiques qui avaient conduit à la constitution de cette classe.

\subsection{Un exemple : l'approche instrumentale}

Nous présentons uniquement les étapes du traitement correspondant à l'approche instrumentale (les résultats complets de cette recherche figurent dans [CNC 00]).

\subsubsection{La grille de lecture}

La partie de la grille correspondant à l'approche instrumentale ( $\$ 2$ et 3 ) comprenait les questions suivantes :

- Le temps est-il évoqué comme variable importante?

- Si oui, comment ce temps est-il situé par rapport au temps des environnements habituels?

- Les contraintes de l'outil pour l'action sont-elles évoquées?

- Les potentialités de visualisation sont-elles évoquées?

- Les potentialités de modélisation sont-elles évoquées?

- Les potentialités de programmation sont-elles évoquées?

- La variable "dispositif d'enseignement intervient-elle dans l'analyse?

- La variable « dimension sociale/individuelle » intervient-elle dans l'analyse?

- La variable « disponibilité de l'outil» intervient-elle dans l'analyse?

- La dimension instrumentation intervient-elle dans l'analyse?

\subsubsection{Les classes analysées}

Le traitement statistique correspondant fournit une partition en 6 classes. Seules les classes 1, 4 et 5 seront décrites ici (les autres, pour des raisons de faible effectif ou de réponses négatives à l'ensemble des questions posées, apportent peu d'information).

L'analyse de chaque classe a été réalisée à partir des traits communs aux articles de la classe et à partir des articles que le traitement fait apparaître au centre de chaque classe.

\subsubsection{La classe 1}


Elle comporte 5 articles français et 9 articles étrangers, dont 6 anglo-saxons (en tout, 8 pays représentés). L'angle d'attaque est clairement l'intégration de l'outil pour l'enseignement. Cette classe est caractérisée par la prise en compte du dispositif, des contraintes et de la dimension sociale. Il peut paraître étonnant que la variable temps ne soit pas toujours prise en compte, alors qu' une grande attention est accordée aux dispositifs d'enseignement. Une hypothèse est que, quand l'outil est continûment disponible, les contraintes de temps sont moins importantes. Le traitement statistique fournit dans cette classe les articles centraux suivants :

- [CHA 98], portant sur l'algèbre (niveau collège américain), étudie en particulier la variable disponibilité : les élèves qui ont eu une disponibilité continue de l'outil acquièrent une meilleure aptitude à l'analyse graphique et symbolique. La disponibilité partielle de l'outil peut entraîner un sentiment de frustration et une attitude plus critique vis-à-vis de la technologie.

- [PRA 97], portant sur la contruction géométrique, met en évidence la nécessité d'une intervention de l'enseignant pour décontextualiser les connaissances.

Les questions posées dans ces deux articles sont différentes : le lien probable entre ces résultats, c'est qu'ils ne sont pas nécessairement très positifs du point de vue de l'apprentissage, justement parce qu'ils font suite à une interrogation et une validation à partir de données recueillies. En cela, ils tranchent avec des articles plus généraux dont les résultats peuvent être d'autant plus positifs qu'ils ne sont pas contredits par une expérimentation comportant des observations sur une durée longue de situations de classes prenant en compte les caractéristiques des environnements.

\subsubsection{La classe 4}

Elle comporte 4 articles français, un article américain et un article australien. Il s'agit essentiellement d'articles français de géométrie. L'angle d'attaque est ici clairement le temps nécessaire à l'intégration des instruments. Ce qui différencie cette classe de la classe 1 est la prise en compte du temps, la non prise en compte des dispositifs d'enseignement.

Le traitement statistique fait apparaître comme article central [GOL 95]. Il évoque les possibilités de transformation continue des figures par le logiciel (géométrie dynamique). Du fait de l'incapacité des élèves à organiser l'exploration, l'auteur s'interroge sur l'effet du logiciel sur la conceptualisation. Il ne pose pas le problème du contrôle de l'usage du logiciel par le professeur. C'est une réflexion personnelle, d'une portée générale pour une problématique d'intégration. 


\subsubsection{La classe 5}

Elle comporte 4 articles français, 2 articles autrichiens, un article anglais et un article américain. L'angle d'attaque est ici la question du temps, appréhendée de façon contradictoire : $50 \%$ des articles estiment que la technologie permet de gagner du temps, $50 \%$ des articles estiment que le contraire est parfois vrai. Les contraintes de l'outil pour l'action ne sont pas évoquées, contrairement aux possibilités de visualisation et de modélisation. La variable dispositif d'enseignement, ainsi que la disponibilité de l'outil et l'instrumentation, interviennent rarement dans l'analyse. Les articles centraux dans cette classe sont les suivants :

- [KUN 98] aborde des idées mathématiques intéressantes sur les transformations géométriques. Les nombreuses difficultés liées au dispositif informatique se transforment en avantages pédagogiques. On peut parler d'une vision apologétique de l'intégration. Notons que les activités informatiques des élèves ne sont pas détaillées.

- [MAY 94] est représentatif d'études comparatives menées sérieusement. Il met en évidence qu'il n'y a pas de différence notoire en ce qui concerne les habiletés calculatoires et mentionne un progrès marginal en ce qui concerne la résolution de problèmes pour le groupe expérimental. La seule différence significative est le développement d'un point de vue critique des élèves du groupe expérimental vis-à-vis de l'utilisation de l'ordinateur comme outil d'apprentissage.

Les résultats de ces articles sont fort différents, nous avons bien deux «profils » parmi les articles de cette classe, correspondant aux deux réponses données relativement au temps : un premier profil «optimiste », un deuxième profil plus critique.

\section{Conclusion et perspectives}

La faible prise en compte de la gestion institutionnelle des savoirs et pratiques instrumentées et de l'analyse des processus d'instrumentation que l'on observe dans la plupart des articles étrangers peut s'expliquer : l'existence d'un programme national en France contraint à dépasser une approche essentiellement cognitive et à porter attention à la dimension institutionnelle. En ce qui concerne les problématiques des articles retenus, dans un premier groupe d'articles, l'amélioration apportée par les EIAH dans l'apprentissage et l'enseignement est présentée comme un postulat, alors que dans le second groupe cette amélioration est questionnée : ces résultats sont tout à fait en cohérence avec ceux de [PEN 96].

Bien que nos réflexions se limitent à l'enseignement des mathématiques, le questionnement et la méthodologie élaborés nous semblent pouvoir être transférés 
sous certaines conditions à d'autres disciplines. Cette étude permet une meilleure compréhension de la portée des résultats d'un article donné, traitant de l'usage d'un EIAH dans l'enseignement. En effet, les différents points abordés dans la première partie de cet article, tels que l'étude des contraintes et des potentialités de l'EIAH en vue de leur exploitation didactique, le suivi des genèses instrumentales, le temps de l'instrumentation lié à la disponibilité de l'outil, l'importance du rôle de l'enseignant en ce qui concerne la gestion du temps et la mise en place de dispositifs viables dans le système éducatif restent fondamentaux pour une problématique de l'usage des EIAH dans l'enseignement des autres disciplines.

Enfin, signalons que, dans les articles présentant un questionnement réel sur l'intégration, l'interaction homme-machine est encore peu étudiée ${ }^{5}$. Cependant, les évaluations de l'usage d'environnements conçus à partir d'une analyse didactique, tels que [JEA 97 ; LUE 00], permettent d'imaginer l'intérêt que pourrait avoir, pour la conception d'EIAH, une analyse didactique de l'usage intégrant différentes approches, donc susceptible de mieux prendre en compte la complexité des phénomènes en jeu.

\section{Références}

[AND 87] ANderson J. R., Boyle C. F., FARell R., Reiser B. J., « Cognitive principles in the design of computer tutors », in Modelling Cognition, P. Morris, John Wiley \& Sons Ltd, 1987, p. 93-133.

[AND 92] ANDERSON J. R., « Intelligent Tutoring and High School mathematics », Actes du colloque ITS' 92, Montreal, 1992, Editions Springer-Verlag, Berlin, p. 1-10.

[ART 95] ARTIGUe, M., " Une approche didactique de l'intégration de l'EIAO à l'enseignement », in Guin D., Py D., Nicaud J.-F. (eds), Environnements Interactifs d'Apprentissage avec Ordinateur, 1995, Paris, Editions Eyrolles, p. 17-28.

[ART 99] ARTIGUe, M, LAGRANGE J .-B., « Instrumentation et écologie didactique de calculatrices complexes : éléments d'analyse à partir d'une expérimentation en classe de 1ère $\mathrm{S} »$, in Guin D. (ed), Actes du colloque Calculatrices symboliques et géométriques dans l'enseignement des mathématiques, 1999, IREM de Montpellier, p. 15-38.

[BAK 99] BAKER M., VRIES E., LUND K ., « Designing computer-mediated epistemic interactions », in S. P. Lajoie, Vivet M. (eds), Actes du colloque AIED'99, 1999, IOS Press, Amsterdam, p. 139-146.

[BAL 94-1] BALACHEFF N., « Didactique et intelligence artificielle », Recherches en Didactique des Mathématiques, vol 14, $\mathrm{n}^{\circ} 1 / 2,1994$, p. 9-42.

[BAL 94-2] BalachefF N., Sutherland R., « Epistemological Domain of Validity of Microworlds. The case of Logo and Cabri-géomètre », in Lewis R., Mendelsohn P. (eds), Actes de IFIP, Lessons from Learning, 1994, North-Holland, Amsterdam, p. 137-150.

[BAR 91] B ARON G.-L., « Quelques réflexions sur l'usage des tuteurs intelligents », in Baron M., Nicaud J.-F. (eds), Actes des deuxièmes journées EIAO de Cachan, 1991, p. 247-251.

\footnotetext{
${ }^{5}$ Les études portant sur l'interaction entre apprenants [BAK 99] ou sur la nature des connaissances mises en jeu dans l'interaction élève-enseignant [LUN 99] ne se situent pas dans une problématique d'intégration.
} 
[BAR 93] BARON M., Robert A., « Métaconnaissances en I.A., en E.I.A.O. et en didactique des mathématiques », Numéro spécial des cahiers de DIDIREM, IREM, LAFORIA \& IBP, 1993, Université Paris VII.

[BAR 96] BARON G.-L. BRUILlARD E., « L'informatique et ses usagers dans l'éducation », Paris, Editions PUF, 1996.

[BOS 99] Bosch M., Chevallard Y., "La sensibilité de l'activité mathématique aux ostensifs », Recherches en Didactique des Mathématiques, vol 19, n 1, 1999, p. 77-124.

[BRU 94] BRuillard E., Vivet M., « Concevoir des EIAO pour des situations scolaires, approche méthodologique », Recherches en didactique des mathématiques, vol 14, $\mathrm{n}^{\circ} 1 / 2$, 1994, p. 274-299.

[BRU 97] BRUILLARD E., «Les machines à enseigner », Paris, Editions Hermès, 1997.

[CHA 98] Chacon P. R., Soto-Johnson H., " The effect of CAI in College Algebra Incorporating both Drill and Exploration », International Journal of Computers in Mathematics Education, vol 5, n 4, 1998, p. 201-216.

[CHE 92] Chevallard Y., «Intégration et viabilité des objets informatiques dans l'enseignement des mathématiques », in Cornu B. (ed), L'ordinateur pour enseigner les mathématiques, 1992, Paris, Editions PUF, p. 183-204.

[CHE 96] Chevallard Y., « La fonction professorale : esquisse d'un modèle didactique », in Noirfalise R. et Perrin M.-J. (eds), Actes de l'école d'été de didactique, 1996, IREM de Clermont-Ferrand, p. 83-122.

[CNC 00] CNCRE, « De l'analyse de travaux concernant les T.I.C. à la définition d'une problématique de leur intégration dans l'enseignement », rapport de recherche, octobre 2000, IREM, Université Paris VII.

[DAG 96] DAGHER A., « Apprentissage dans un environnement informatique : possibilité, nature, transfert des acquis », Educational Studies in Mathematics, vol 30, 1996, p. 367398.

[DEF 00] DefouAD B., «Etude de genèses instrumentales liées à l'utilisation de calculatrices symboliques en classe de première $S$ », Thèse de doctorat, Université Paris VII, 2000.

[GOL 95] GoldENBERG P., « Ruminations about dynamic imagery (a strong plea for research) », in Sutherland R., Mason J. (eds), Exploiting Mental Imagery with Computers in Mathematics Education, 1995, Berlin, Editions Springer-Verlag, p. 202-224.

[GUI 91] GuIN D., « Modélisation de la démonstration géométrique dans Geometry Tutor », Annales de Didactique et de Sciences Cognitives, vol 4, 1991, p. 2-37, IREM de Strasbourg.

[GUI 95] GuIN D., «A Cognitive Analysis of Geometry Proof Focused on Intelligent Tutoring Systems », in Laborde J.-M. (ed), NATO Series F, Intelligent Learning Environments: The Case of Geometry, Editions Springer-Verlag, vol 117, 1995, p. 82-93.

[GUI 99] Guin D., Trouche L., « The complex process of converting tools into mathematical instruments: the case of calculators », International Journal of Computers for Mathematical Learning, vol 3, n 3, 2000, p. 195-227.

[GUI 00] GuIN D., Trouche L., « Thinking of new devices to make viable symbolic calculators in the classroom », Actes de PME 24, Hiroshima, juillet 2000, vol 3, p. 3-9.

[JEA 97] Jean S., Delozanne E., Jacobini P., Grugeon B., « Conception, réalisation et évaluation d'interface en EIAO, l'exemple de PEPITE », in Baron M., Mendelsohn P., Nicaud J.-F. (eds), Environnements Informatiques d'Apprentissage avec Ordinateur, 1997, Paris, Editions Hermès, p. 37-48. 
[KOE 97] KoEdinger K. R., Anderson J. R., « Intelligent Tutoring Goes To School in the Big City », International Journal of Artificial Intelligence in Education, vol 8, 1997, p. 30-43.

[KUN 98] KUNTZ G., «Une transformation oubliée qui sort de l'ordinaire : l'inversion », Repères-Irem, vol 30, 1998, p. 23-38, Topiques-Editions.

[KUT 94] KUTZLER B., " The future of Teaching Mathematics », International Derive Journal, vol 1, n¹, 1994, p. 37-48.

[LAB 99] LABORDE C., « Vers un usage banalisé de Cabri-géomètre avec la TI-92 en classe de seconde : analyse des facteurs de l'intégration », in Guin D. (ed), Actes du colloque Calculatrices symboliques et géométriques dans l'enseignement des mathématiques, 1999 , IREM de Montpellier, p. 79-94.

[LAG 99-1] LAGRANGE J.-B., " Complex calculators in the classroom: theoretical and practical reflections on teaching pre-calculus », International Journal of Computers for Mathematical Learning, vol 4, $n^{\circ} 1,1999$, p. 51-81.

[LAG 99-2] LAGRANGE J .-B., «Les instruments de calcul formel », in Bailleul M. (ed), Actes de l'Ecole d'Eté de Didactique des Mathématiques, 1999, IUFM de Caen, p. 214-234.

[LUE 98] Luengo V., BALAChefF N., « Contraintes informatiques et environnements d'apprentissage de la démonstration en géométrie », Sciences et Techniques Educatives, vol 5, n 1,1998 , p. 15-45.

[LUE 00] LUENGO V., « Analyse et prise en compte des contraintes didactiques et informatiques dans la conception et le développement du micromonde Cabri-Euclide », Sciences et Techniques Educatives, vol 6, n², 2000, p. 241-268.

[LUN 99] LUND K ., BAKER M., « Teacher's collaborative interpretations of student's computer-mediated collaborative problem solving interactions », in S. P. Lajoie, Vivet M. (eds), Actes du colloque AIED'99, 1999, Amsterdam, IOS Press, p. 147-154.

[MAY 94] MAYES R., «Implications of Research on CAS in College Algebra », International Derive Journal, vol 1, n², 1994, p. 21-38.

[PEN 96] Penglase M., Arnold., « The Graphics Calculator in Mathematics Education: a Critical Review of Recent Research », Mathematics Education Research Journal, vol 8, 1996, p. 58-90.

[PRA 97] PRATT D., Ainley J., « The Construction of Meanings for Geometric Construction: Two Contrasting Cases », International Journal of Computers for Mathematical Learning, vol 1, n 3, 1997, p. 293-322.

[RAB 99] RABARDEL P., «Eléments pour une approche instrumentale en didactique des mathématiques », in Bailleul M. (ed), Actes de l'Ecole d'Eté de Didactique des Mathématiques, 1999, IUFM de Caen, p. 203-213.

[TRO 96] Trouche L., « Masques », Repères-Irem, vol 23, 1996, p. 43-64, TopiquesEditions.

[TRO 00] TROUCHE L., « La parabole du gaucher et de la casserole à bec verseur : étude des processus d'apprentissage dans un environnement de calculatrices symboliques », Educational Studies in Mathematics, vol 41, 2000, p. 239-264.

[VER 96] Vergnaud G., «Au fond de l'apprentissage, la conceptualisation », in Noirfalise R., Perrin M.-J. (eds), Actes de l'Ecole d'Eté de Didactique des Mathématiques, 1996, IREM de Clermont-Ferrand. p. 174-185.

[VIV 90] VIVET M., «Uses of ITS: Which Role for the Teacher ? », in E. Costa (ed), New directions for Intelligent Tutoring Systems, NATO Series F, Berlin, Editions SpringerVerlag, vol 91, 1990, p. 171-180. 